\title{
Pilot Data on the Performance of Vibrotactile Actuators for Use with Assistive Devices
}

\author{
Christiane Courtemanche, Mojtaba Ahmadi \\ Carleton University \\ 1125 Colonel By Drive, Ottawa, Canada \\ christiane.courtemanche@carleton.ca; mojtaba.ahmadi@carleton.ca
}

\section{Extended Abstract}

As human-machine interfacing becomes increasingly common, the importance of biofeedback optimization escalates. Many machine-user interfaces currently require the use of biofeedback; for instance, fall prevention and posture improvement devices often make use of vibrotactile biofeedback to communicate to the user the necessary body motions to regain balance or maintain a proper posture, thereby enhancing or replacing the body's natural position biofeedback system and closing the biofeedback loop. Vibrotactile biofeedback could also be combined with an exoskeleton or a powered orthotic for the aforementioned purposes in users that require machine assistance to vary their body position. Although these devices employ biofeedback, there exists no thorough research on the optimization of vibrotactile biofeedback parameters such as actuator location on the body, actuator type or information coding method. Some studies researched the implications of one or two of these parameters, but no study has considered all 3 parameters. This study aims to optimize biofeedback by minimizing the user's reaction time and discomfort, and by enhancing the ability to correctly identify the tactor activated and its intensity. In order to determine the optimal biofeedback configuration the tactor type, information coding method and actuator location on the user's body were varied and the combination showing the best overall results was selected as the optimal biofeedback configuration.

This study was conducted in two parts to decrease the length of tests performed on 3 healthy participants. Part I compared and characterized the properties of two tactor types (Eccentric Rotating Mass (ERM) and Linear Resonant Actuator (LRA)) attached to different body site types (skin-covered muscle, lipid and bone). The primary outcome measures for this study were the vibration frequency, acceleration amplitude and actuator displacement. Secondary outcomes were the ability to detect actuator activation and the inter-participant variation in vibration properties. Part II determined the ideal actuator location by comparing the performance of a 4-actuator system for 5 body locations (neck, shoulder, wrist, waist and ankle) and 2 information coding methods (vibration intensity and duration). Primary outcomes are comfort level, reaction time, activated tactor location and intensity identification accuracy. The secondary outcome will be the accuracy in predicting sensation using tactor vibration characteristics.

Feedback using the ERM was unanimously better detected by participants. As expected, the ERM's vibration frequency increased with the voltage supplied, whereas the LRA's vibration frequency remains constant; regardless of the changes in frequency with voltage, the ERM remains the most easily discernible biofeedback device. Furthermore, acceleration amplitude, vibration frequency and displacement were poor indicators of the wearer's ability to sense vibrotactile biofeedback; only displacement in the main axis of motion normalized to the participant's BMI shows promise in predicting vibration detection by the wearer. For the ERM, the prediction specificity and sensitivity were $39 \%$ and $96 \%$, respectively, whereas for the LRA they were $85 \%$ and $84 \%$. Furthermore, when comparing frequency, acceleration amplitude and displacement across participants, statistically significant differences $(P$ values $<0.001)$ were observed, however, these values are not effectively significant because the differences between participants mean values were negligible.

\section{References}

[1] M. Janssen, et al., "Salient and placebo vibrotactile biofeedback are equally effective in reducing sway in bilateral vestibular loss patients," Gait \& Posture, vol. 31, no. 2, pp. 213-7, 2009.

[2] B. C. Lee, et al., "The effects of actuator selection on non-volitional postural responses to torso-based vibrotactile stimulation," J. Neuroeng. Rehab., vol. 10, no. 1, 2013.

[3] K. H. Sienko, et al., "Assessment of vibrotactile feedback on postural stability during pseudorandom multidirectional platform motion," IEEE Trans. Biomed. Eng., vol. 57, no. 4, pp. 944-52, 2010.

[4] C. Wall, et al., "Balance prothesis based on micromechanical sensors using vibrotactile feedback of tilt," IEEE Trans. Biomed. Eng., vol. 48, no. 10, pp. 1153-61, 2001. 